\title{
On the Security of Digital Tachographs *
}

\author{
Ross Anderson \\ Cambridge University Computer Laboratory \\ Pembroke Street, Cambridge CB2 3QG \\ rja14@cl.cam.ac.uk
}

\begin{abstract}
Tachographs are used in most heavy vehicles in Europe to control drivers' hours, and for secondary purposes ranging from accident investigation and environmental enforcement to the prevention of fraud. Their effectiveness is under threat from increasing levels of sophisticated fraud and manipulation. We examine this in the context of recent EU proposals to move to smartcard-based tachograph systems, which are aimed at cutting fraud and improving the level of enforcement generally. We conclude that the proposed new regime will be extremely vulnerable to the wholesale forgery of smartcards and to system-level manipulation, which in turn could lead to a large-scale breakdown in control. We present this as an interesting and important problem to the security community.
\end{abstract}

Keywords: smartcard, tampering, fraud, tachographs

\section{Introduction}

Vehicle accidents where the most likely cause was the driver falling asleep at the wheel account for about $16 \%$ of the total on all UK roads, and about $23 \%$ on motorways [11]. Death or serious injury is significantly more likely than in other types of accident, probably because of the greater speed on impact [26]. Consistent figures have been reported from the USA, Germany, Israel and Sweden [11]. By comparison, vehicle accidents where alcohol is a significant contributory factor range from $3.1 \%$ in the UK through $4.9 \%$ in Sweden and $9.5 \%$ in Germany to $14.1 \%$ in Finland [28].

Heavy commercial vehicles are a particular problem as they can do much more damage in a crash. Although they are involved in only $6 \%$ of serious accidents, these include $16 \%$ of fatal accidents.

In Europe, the principal control on the hours worked by heavy vehicle drivers is the tachograph - a device fitted behind the speedometer which logs the vehicle's speed, distance and mode of work on a waxed paper chart, in the centre of which the driver must write his name, starting location, vehicle number,

* This paper reports research funded by the Department of the Environment, Transport and the Regions during 1997-98. 
date and odometer reading before commencing his journey. Tachograph use is mandatory for most heavy trucks in the European Union, and about half the bus and coach fleet. Drivers must carry with them their charts for the current week and the last driving day of the previous week, after which they must be stored at the employer's premises for one year.

Although the system was introduced to control drivers' working hours, it has since acquired a number of other uses. The police find tachograph charts helpful in investigating accidents and other offences, such as unlicensed toxic waste dumping, while many operators use them to prevent theft of fuel by drivers (which can amount to 5,000 ECU per vehicle per year otherwise) [24]. Almost a third of tachograph charts are already scanned by fleet operators or bureaux and fed into fleet management systems for this purpose; these operators would welcome digital tachographs. However, safety remains the main purpose of the system.

About 200 fatalities a year result from sleep-related accidents involving heavy vehicles in Britain; perhaps half of these might be prevented by draconian enforcement of driving hours regulations, while the total might be doubled by a breakdown in control. In countries with lax enforcement, the prospective gains should be higher and the potential losses lower. In total, a uniformly high level of control might save the EU a thousand lives a year and a sum in the low billions of ECUs, while a breakdown might have additional human and economic costs on the same scale.

\section{Tachograph Tampering and Fraud}

There is therefore great concern at a growing wave of tachograph fraud and tampering, by which both drivers' hours and speed regulations are flouted on a large scale. Most of this fraud is motivated by economic pressures on vehicle operators, and might be reduced by better tachograph systems.

Good security engineering requires a detailed understanding of threats. We therefore list here the main techniques used at present for tachograph fraud and tampering. Our figures for their relative prevalence come from a recent survey by the UK Vehicle Inspectorate of convictions for tachograph offences [9]: the sample size was 1060 , made up of 854 convictions of drivers and 206 convictions of operators.

1. Most frauds do not involve tampering but exploit procedural weaknesses. These accounted for $68 \%$ of driver and $71 \%$ of operator convictions.

- A very common fraud is 'ghosting' - manipulating tachograph charts so that there appear to be more drivers than there actually are. For example, a company with premises in Dundee and Southampton should have four drivers in order to operate one vehicle per day in each direction; the distance is about $800 \mathrm{~km}$ and the journey takes about 10 hours which is illegal for a single driver to do without an overnight rest. 
The standard fiddle is to have two drivers, who meet en route at Penrith, change trucks, and insert new paper charts into the tachographs. The driver from Southampton now returns there with the vehicle from Dundee. When stopped and asked for his charts, he would show the current chart from Penrith to Southampton, the previous day's for the leg from Southampton to Penrith, the day before's for Penrith to Southampton, and so on. In this way he would give the false impression that he spent every other night in Penrith and was thus legal. This practice, of swapping vehicles halfway through the working day, is widespread [21].

- Much casual deceit involves very simple manipulation, such as altering the clock to simulate a rest period, inserting a fresh tachograph chart and hiding the old one, forging a chart by hand (perhaps with the help of compasses or bottle tops) or even driving with no chart at all and hoping to produce an old one by sleight-of-hand if stopped [29]. These tricks are likely to be detected if the vehicle is stopped by an alert officer; they are often used when a normally honest driver is delayed and is under pressure to deliver a load rather than take an unscheduled break.

- More sophisticated procedural frauds include 'forgetting' to write the date on the chart centre field, representing a hitch-hiker as a co-driver, using a chart for a $140 \mathrm{~km} / \mathrm{h}$ tachograph in a $125 \mathrm{~km} / \mathrm{h}$ device, filling completely fictitious centre field details, and representing the start point of the journey as an obscure village [21]. They often involve collusion with the operator, as with ghosting: often, when the operator is ordered to produce charts and supporting documents such as pay records, ferry tickets etc., his office will conveniently burn down.

2. The next largest category of fraud involves tampering with the supply to the tachograph instrument, including interference with the power and impulse supply, cables and seals. Offences involving electronic tachographs amounted for $23 \%$ of driver convictions and $18 \%$ of operator convictions; offences involving the older mechanical instruments added another $2 \%$ and $3 \%$ respectively.

- Such frauds often involve collusion with fitters. Electronic tachographs get their input from a sensor in the gearbox, which sends electrical impulses as the prop shaft rotates, and a common attack is to unscrew the sensor about $2 \mathrm{~mm}$. This causes the impulses to cease, as if the vehicle were stationary. To prevent this, gearbox sensors are fixed in place with a wire and lead seal. Fitters are bribed to wrap the wire anticlockwise rather than clockwise, which causes it to loosen rather than break when the sensor is unscrewed. The fact that seals are kept by workshops rather than by individual fitters complicates prosecution.

- Some determined offenders fit a switch into the cable so that the input can be drawn either from the real gearbox sensor or from an additional sensor that is mounted under the driver's seat rather than being rotated by the gearbox [21]. At least one operator has had all its vehicles wired to interrupt impulses on demand. 
- However the two most common techniques are very simple: to insert an earthing wire into the cable, thus shorting out the impulses, and to replace the tachograph fuse with a blown one. The incidence of the former attack has been reduced recently by a switch to armoured cables ${ }^{1}$, but blown fuses continue to provide a plausible excuse [21].

3 . The third category of fraud is tampering with the tachograph head itself (the unit mounted behind the speedometer). $4 \%$ of driver offences, and $5 \%$ of operator offences, are ascribed to this kind of abuse of the older mechanical tachographs, with a further $1 \%$ and $2 \%$ respectively booked for attacks on the newer electronic devices.

Most tachograph head tampering involves miscalibration, which is often performed with insider assistance. Tachograph heads contain potentiometers or switches with which the radius of the road wheels can be set, thus translating impulses into distance and impulse rates into speed. Corrupt fitters often set these to indicate some $90 \%$ of the actual road speed. Drivers may also break seals and change the calibration directly [22], and steam cleaning often destroys seals anyway thus providing a good excuse. In some cases, drivers have broken seals and repaired them invisibly $[10,21]$. The current seals are easy for skilled persons to defeat, and their knowledge is spreading rapidly [14]. Work needs to be done urgently in this area.

The remaining attacks tend to exploit vulnerabilities discovered by chance, knowledge of which spreads more rapidly among drivers than among policemen. They include:

- in many devices, one can bend the styli that write on the chart and thus falsify the recorded speed [22];

- one device can be caused to register zero speed by inserting a piece of wire into the tachograph head. This shorts the circuitry to earth without breaking the seal. In another device, an earth wire can similarly be inserted into a sealed cable joint. These wires can be pulled out in a second if the vehicle is stopped by police [22];

- the earlier electronic tachographs had the power for the impulse generators and head motors wired through the ignition circuit to minimise battery drain, while the clock and lighting circuits come directly from the battery. However diesel engines once started can run with the ignition off, in which case the device is frozen. The speed stylus remains roughly where it was, while other traces will indicate a vehicle at rest;

- in the following generation of electronic tachographs, the clock and the motors operated at different voltages, and the device could be frozen by reducing the supply voltage. In one model, the overspeed warning light helpfully came on at just the right voltage [21];

- with some models, one could wire up a flasher unit to interrupt the supply voltage and thus reduce the rate at which the chart table turned;

- when features to detect supply voltage interruption were introduced ${ }^{2}$, not all of them were well engineered. In one device, an alarm causes the

${ }^{1}$ Commission Regulation 2470/95

${ }^{2}$ as a result of Commission Regulation 3314/90 
speed stylus to strike downwards, which has no effect if the vehicle is stationary when power is resumed. In another device, the alarm can be suppressed by connecting two generally unused terminals together [21];

- with one model, the driver can press down on the centre of the speedometer plastic and prevent the needle from moving when he accelerates from (say) 30 to $60 \mathrm{~km} / \mathrm{h}$. As the assembly is driven by a stepper motor, both the speedometer and the underlying tachograph will register $30 \mathrm{~km} / \mathrm{h}$ less than the true speed until this speed next drops below $30 \mathrm{~km} / \mathrm{h}$.

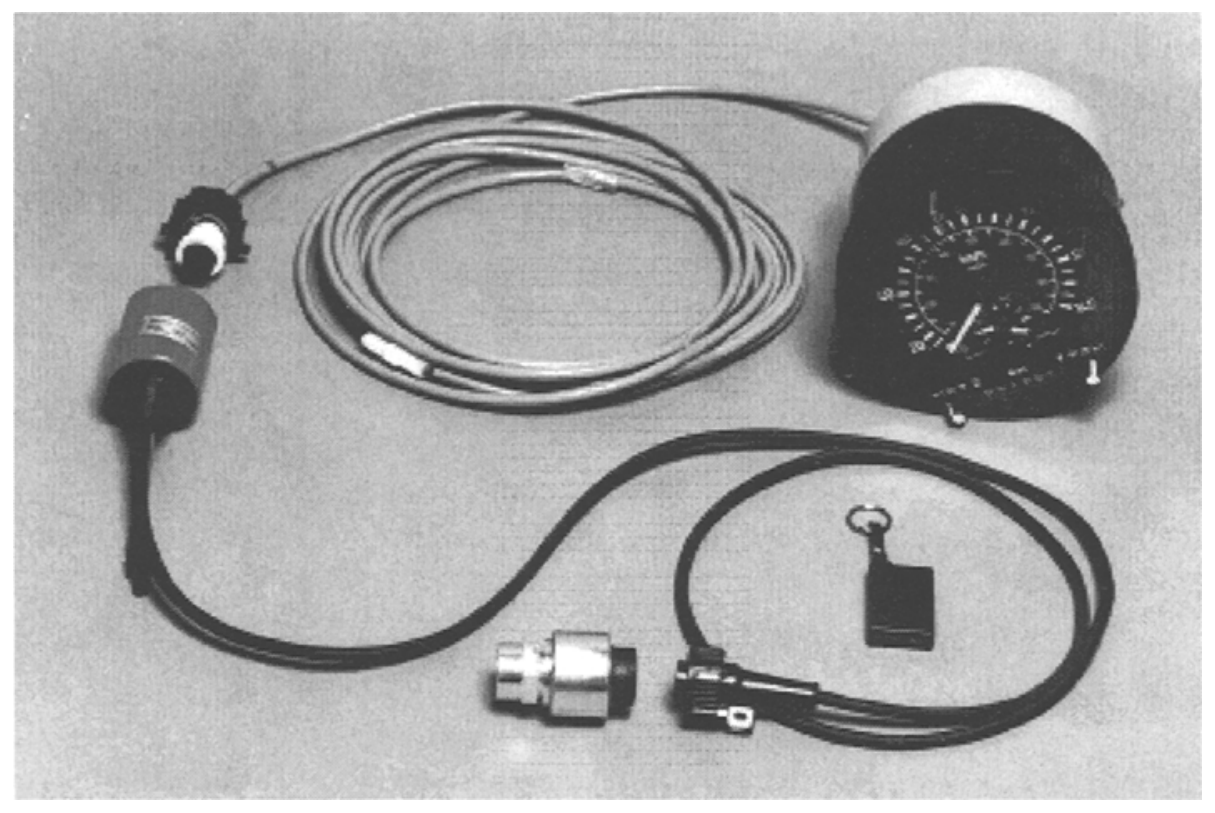

Fig. 1. A tachograph with an interruptor controlled using a radio key fob.

4. The state of the tampering art is a radio controlled interruptor device depicted in figure 1. This is a red plastic cylinder bearing the inscription 'Voltage Regulator - Made in Japan' but which is actually inserted into the tachograph cable and controlled by the driver from the cab using the remote control key fob. A first press causes the indicated speed to drop by $10 \%$, a second press cuts it by a further $10 \%$, a third press causes it to fall to zero, and a fourth causes the device to become inactive so that the tachograph and speed limiter return at once to proper operation. Such devices are extremely hard to find as they can be hidden at many different places in the truck's cable harness (miniature versions are even found inside the tachograph head itself). Police officers who stop a speeding truck equipped with such a device, and cannot find it, have difficulty getting a conviction 
as the sealed and apparently correctly calibrated tachograph contradicts the evidence from their radar or camera.

Only six convictions - five driver and one operator - fall into the category of 'Radio controlled devices etc' but intelligence suggests that their use is becoming widespread; the lack of convictions is due to the difficulty of detection.

As with many of the so-called 'victimless' crimes that are usually detected only by enforcement action, hard figures on the extent of tachograph manipulation are not readily available. In any case the extent and methods of fraud vary by country. In countries with poor enforcement, drivers may simply not use charts at all (industry sources named two member states with negligible chart sales).

However, the consensus of informed people in the UK is that maybe 5-10\% of drivers are persistent offenders and about half offend occasionally. The persistent offenders tend to be associated with certain operators, some $20-40 \%$ of whose vehicles turn out to have signs of past tampering when examined closely at inspection stations. These signs include seal defects, calibration faults and suspiciously loose wiring [30]. The significance of this is that well funded attempts to defeat future tamper proofing systems can be anticipated; at least one of the suspect operators has over 500 trucks.

Tachograph tampering brings secondary safety hazards, in that most heavy vehicles must now be fitted with speed limiters, which are usually driven from the tachograph head. So drivers who wish to exceed the speed limit may tamper with the tachograph, and drivers who tamper are tempted to speed as well. Speed limiter tampering is even more widespread than tachograph tampering (as drivers can also attack the limiter, and the cable between it and the tacho head, in various ways). Unfortunately, the introduction of limiters has caused some truck makers to de-rate tyres and other systems, making speeding significantly more hazardous.

One might think that as vehicle systems become more integrated, using standards such as CANBUS, tampering would decrease because unauthorised modifications to systems can have a side-effect on safety. Experience shows that this hope is vain. One truck ran its antilock braking system from the same circuit as the tachograph, so that a driver who replaced the fuse with a blown one would disable his ABS. Many drivers still replaced the fuse.

It might also be thought that the situation has been improving over time, with more recent tachographs being more resistant to tampering. This is not the case; the move from mechanical to electronic equipment led to a tenfold rise in convictions for tampering with the supply. Older devices used rotating wire cables and an attempt to physically jam the odometer caused gear teeth to strip or the cable to shear; the move to electrical impulses was a large setback for enforcers as it enabled odometers to be jammed (which in turn made other frauds easier), while cable earthing and then interruptors opened up a whole new 
set of attacks. Attempted improvements just made the frauds more complex and difficult to detect [21].

It is now generally agreed that the cable from the sensor to the tacho head should be protected by cryptography. But there is still no agreement about how, and it is unclear how unique crypto keys can be loaded safely into the sensor and the head when the fitters are in the pay of the attacker. (In fact, one supplier has proposed loading the same crypto key into every sensor unit.)

A further set of problems comes from the EU member states' different operational models. For example, in the UK much of the enforcement is done by roadside checks, while in the Netherlands it is mostly at operator premises. In addition, a variety of computer systems have been developed to support enforcement, and there are also a number of fleet management systems which are used, as noted above, to prevent theft of fuel by drivers. The actual tachograph is thus a component of many different enforcement and management systems.

\section{The Tachosmart Projects}

This is the background to the emerging consensus in Europe for action at the Community level to improve the dependability of drivers' hours recording $[7,29]$.

The EU tachograph market is dominated by two companies, VDO Kiezle of Germany and TVI of Britain, with about $75 \%$ and $25 \%$ respectively of an equipment market worth about $100 \mathrm{MECU}$ per annum. Sales made by analysis bureaux, fitters, calibrators, suppliers of consumables and spare cables, etc., make up the total tachograph market value to some 300-400 MECU per annum - about an order of magnitude less then the annual economic cost of sleep related accidents.

So a change to the system can be motivated by enforcement considerations, and the EU has funded a 'Tachosmart' project to develop a more tamper resistant electronic replacement for the current chart-based systems. There have been three phases so far, and Tachosmart 3 resulted in prototype devices built by manufacturers in five different member states [27].

The prototypes use a smartcard rather than a chart to personalise the equipment. This introduces a significant memory constraint. The cards used in the prototype have $8 \mathrm{~K}$ EEPROM of which a little over $5 \mathrm{~K}$ is available for driver record keeping; production cards are planned to have twice as much memory. However, paper charts keep speed history with a resolution, under microscopic examination, of 1 second - equivalent to a capacity of several tens of kilobytes per chart. Recording 28 days' (or even 14 days') driving activity on a commodity smartcard is thus out of the question. Thus a smartcard based tachograph must either have auxiliary memory, store a much compressed record, or both. 


\subsection{The proposed solution}

The proposed solution is that a memory in the vehicle unit will retain 365 days' drivers' hours data, plus a speed history for the last 24 hours. The driver card will have memory for 28 days' driving hours, but no speed history. There will also be cards for the vehicle operator, vehicle inspectors, calibration stations and fitters which will give differing kinds of access to the data in the vehicle unit. The vehicle operator, for example, will be able to download complete data for integration into a fleet management system. Some of the interfaces, including those between the various types of card and the vehicle units, will be standardised across the EU.

In the prototype systems, the impulses are generated as before in a gearbox sensor, and passed to the vehicle unit (the means of protecting the impulses from tampering are left to the manufacturer). They are converted in the vehicle unit into a speed history which is retained in memory for 24 hours and may be downloaded using an operator or inspector card. The vehicle unit can also store more alarms than the card; typically 10-25 of each type. It will normally have one or two cards in it, and in normal operation these will be drivers' cards. It will then associate driver's and if appropriate co-driver's hours to them in its internal log. A much compressed history of drivers and hours will be kept for 365 days.

Cards (of whatever type) are authenticated to the tachograph by a bidirectional challenge-response using keys that are common throughout the system; data exchanges between tachographs and the cards carried by drivers and inspectors are claimed by the security specification to employ digital signatures [8]. However, the specification of the card contents does not contain enough room for signature keys $[15,16]$. We conclude that, as often in the smartcard industry, 'digital signature' or 'data signature' actually means a message authentication code computed using a common secret. This leaves it unclear how signatures are to be verified in the software of external systems, and how the system will interact with German digital signature law.

Key material is loaded into drivers' cards by a national card issuing authority. The vehicle units are manufactured with embedded secret keys that are common throughout the system, and every time they are calibrated, new signature keys and certificates are loaded from a calibration card using the common secret for authentication. The protocols used are not specified in sufficient detail for close analysis.

In theory, the driver's card is retained in the instrument during driving (though ensuring this is harder than it looks), and at the end of the trip it is updated with a signed record of working hours plus the last three alarms (overspeeds, tampering events, etc) if any. The card can retain up to 28 days' records, depending on driving conditions.

At any time, an inspector can request a print-out from the vehicle unit in one of two ways. If he has a control card, he can obtain a signed copy of the contents 
of the tachograph memory transmitted to a lap-top over a serial link [17]; if not, the driver can print out appropriate data from the vehicle unit's memory and hand it to the inspector.

\subsection{Anticipated problems}

The simultaneous operation of vehicle fleets with paper charts and driver cards is expected to lead to serious enforcement problems. In cases involving ghosting, it is predicted that the operator will have one new vehicle that uses a card and an older one that uses charts. (This is expected to raise the price of older vehicles and depress new vehicle sales - an effect already seen with previous changes in regulations, not just for tachographs but also for speed limiters and emissions.)

Conscientious operators will buy systems that will enable downloaded digital tachograph data to be merged with data from scanned analogue charts, fuel purchases, drivers' overtime etc. But, as noted above, there is no EU requirement for such systems. Some countries are considering making them mandatory at the national level, with controls mirroring those proposed for cryptographic systems in general [4] in that large companies would be trusted to run their own systems while the smaller operators would have to use an approved bureau service. This would extend the current system whereby the workshops of large, trusted operators may be approved as tachograph fitters while small operators must use third parties.

In countries where downloading is not mandated, the theory is that drivers would take paper printouts from any digital tachographs they used and keep them along with the paper charts from any analogue devices. This would make enforcement harder; the printouts from the Tachosmart prototype systems are much easier to forge than charts are. It is also unclear how operators could discharge their legal duty to maintain records of drivers' hours [7], as the vehicle units containing this information could be distributed all over Europe and beyond.

There will be serious consequences for competition and the internal market. From July 1998, the EU will have unrestricted cabotage - drivers from one member state will be able to carry goods in another state. The current wide variation in tachograph enforcement does not pose a competitive issue so long as the primary control is carried in the vehicle. However, if Tachosmart moves the primary control to the computer system of the vehicle operator or his bureau, and this control is only effective in some countries, then drivers from other countries will have a competitive advantage which will exert downward pressure on the quality of control everywhere. This will be further complicated by the fact that there are vehicle operators with depots in (say) Belgium and Holland, and who operate a given vehicle out of both of them depending on the day of the week; there are also operators who share vehicles. Even more serious problems may come from non-EU vehicles. The proposals are silent on how the control factors, from card issue onwards, will be managed in that case. 
The second set of anticipated problems comes from the loss of detailed redundant data. At present, fraud and accident investigation depends on comparing the speed and distance traces against the claimed journey end points and against other documents such as delivery notes and ferry tickets. In the digital system, speed history will be retained in the vehicle unit for only 24 hours, and if not downloaded will be lost; while journey end points will only be logged at the granularity of a region, as a keyboard to enter the names of towns would be expensive.

It is not clear how the inspector is to do his job in a digital environment. The detailed, redundant data on the chart often provides inspectors with grounds for suspicion to justify a detailed examination. However digital systems either indicate a violation or do not. Thus while the older analogue systems degrade gracefully under attack, digital systems fail abruptly. This means that a higher level of tamper resistance is needed in the digital environment, to which we will return below. As for the use of additional inputs, the draft regulations permit countries to require inputs from GPS for domestic vehicle, if they wish ([7] article 15 clause 5a). However the Tachosmart standards do not support this - there appears to be no provision for a GPS interface, despite the regulations permitting individual member states to require the use of GPS. If GPS units are not connected to the tachograph but carried as separate equipment, then many of their potential benefits will be lost.

The third problem set concerns reliability. Smartcards started out as bank cards, designed to be used several times a week in cash machines; when they are used heavily such as in building access control and transport ticketing - and especially in grimy environments - common models experience failure rates of up to $7 \%$ per annum. This is not compatible with the expected 5 year card lifetime and 10,000 hour mean-time-to-failure [15], and would be onerous on companies and workshops given that cards are to be issued centrally with replacement taking as much as two weeks [18].

This will facilitate simple frauds by drivers and operators. By destroying a card (e.g. by applying mains electricity) a driver can eliminate a problematic record, and under the regulations he will be allowed to drive for 15 days without a card. Such card-destruction attacks have been perpetrated on bank smartcard systems in the UK, France and elsewhere; by forcing the system back on less secure stand-in procedures many avenues of abuse are opened up. One UK bank has had to open a specialist laboratory to examine failed smartcards presented by customers.

Other reliability issues impinging on security include the failure rate of printers in a commercial vehicle environment.

Many of these objections can be overcome by changes to the details of the specification. However there is a much more fundamental problem with the Tachosmart concept and that is the belief that smartcards are tamper-proof. 


\section{Smartcard Security Issues}

The draft regulations state that 'the total system, including the connections to the speed and distance sensor, must be tamperproof' ([7] p 27). This cannot be achieved given the card technology currently available in Europe.

For years, smartcard vendors claimed that their products were tamperproof, or as nearly so as made no difference. In the last few years, this claim has been demolished by a large number of attacks on pay-TV and on other systems.

The state of the tampering art is constantly evolving. A number of historical attacks are described in [2], together with the techniques used by pirates in 199496. The state of the art in early 1998 is somewhat more advanced; professional pirates now use microscopes fitted with lasers and microprobes to extract card data quite rapidly. One technique is to fit a probe to the line that controls the instruction latch, and use this to prevent new instructions being loaded from the bus. Now when the card is clocked there will be no jump instructions, and all the words in memory will appear on the bus in sequential order. A second microprobe is then used to recover the memory contents from one bus line at a time.

The laser is used to remove the passivation layer from the card surface over the feature to be probed; this avoids removing the whole layer, which may set off an alarm in some card designs. It also creates a depression in which the microprobe will lie stably.

The cost of the laboratory equipment needed to perform this attack is about $150,000 \mathrm{ECU}$, though second-hand equipment is much cheaper. The equipment is also available at many university laboratories, and at least one EU university teaches chipcard breaking techniques to undergraduates as part of their course work [3].

The effect of attacks, carried out both by students and by professional pirates, has been to force pay-TV operators such as BSkyB to change their entire card base about once a year, moving each time to a new technology of card with ever more expensive tamper resistance mechanisms. To date, the pirates have always managed to keep up; an example of the Sky series 9 card, opened with hand tools and yet still functional, can be seen in [2].

The arms race between attackers and defenders is expensive; for example, the technology supplier to BSkyB has seen its research department grow from 60 to 200 people over the past four years, while at a recent trial of a card forger, BSkyB claimed that forgery cost them $£ 30 \mathrm{~m}[13]$. Yet there is still no real breakthrough in tamper prevention, and senior scientists at some large semiconductor makers expect none, for reasons discussed in [2].

Attacks have mostly taken place on systems where universal secrets are stored in cards, and where the compromise of these secrets can enable cards to be forged and sold for mass use (such as pay-TV). They have not been widespread on cards that store only individual secrets and where forgery can bring only limited benefits (such as the SIM cards used in mobile phones). 
The system prototyped in the Tachosmart 3 project falls squarely into the former category. The workshop, operator and inspector cards contain only common secrets and so we expect that they will be rapidly duplicated and sold through the channels currently used to market interruptors (such as ferry terminals and transport cafes). The workshop card will enable alterations to be made to tachograph parameters. The calibration cards, if required after such changes (the specification is vague), contain a list of keys plus a common secret for authentication. These cards will also be duplicated as they will allow speed limits to be changed.

The workshop card can clear the tachograph memory and the calibration card can then be used to re-load keys. If a forged calibration card were used for initialisation of the device in the first place, then the original keys could be re-loaded thus causing an embarrassing record to vanish without trace.

If, as appears in $[15,16]$, the 'data signatures' do not really use asymmetric cryptography but message authentication codes (MACs) computed with shared secrets, then once these common secrets become known, the system will be completely broken. Pirates will be able to manufacture any type of card and forge any type of record.

We will now look in more detail at the likely effects of attacks under various assumptions.

\section{What Will Go Wrong}

If we now work down the list of existing frauds, we can get a rough idea of the effect of introducing the proposed technology in its present form.

1. The initial attraction of the smartcard-based digital tachograph was that it might stop ghosting which together with other chart manipulations amounts for most UK convictions.

However, this would only be the case if (a) digital devices were tamperproof (b) cards could not be forged or duplicated and (c) the entire EU vehicle park were retrofitted with digital devices. None of these is likely to be the case. Retrofitting a vehicle with a tachograph other than of the original type is expensive, as it involves custom engineering; one vendor reported 19 replacement sales last year against 20,000 units repaired. Yet in the absence of retrofit, ghosting will be easy even without system penetration; the operator will just use one digital and one analogue truck.

Attacks involving simple manipulation will become much easier as drivers will be able to cause tachograph malfunctions in many ways (mains electricity in the card, electrical contact failure, sand in the printer, ...). The digital tachograph is much like a burglar alarm in that the attacker only has to destroy confidence in it, by making it appear to be unreliable, in order to defeat it. The lessons from attacks on digital burglar alarms [23] should be studied carefully. 
Frauds in which the operator colludes with drivers and covers up for them by destroying records in the event of an investigation will become more common if record destruction involves only a 'disk crash' on a PC rather than burning down part of the premises as at present. In addition, we expect that the larger rogue operators will invest in manipulating cards. If operators are allowed to keep records on paper, the printouts from vehicle units will be easy to forge, and this may be the method of choice for one-man operators where downloading is not compulsory.

2. Attacks on the sensor seal are not tackled by the proposals, and although new systems are supposed to protect the signalling, this is left to individual vendors with no standards being set. The one unit we examined sent conventional unprotected pulses plus a second channel of encrypted pulses. The latter is used by the tachograph, but only the former is available to the speed limiter. So an interruptor could still be fitted between the tacho head and the limiter.

The simultaneous entry into the market of several new vendors with no experience of the industry will be an aggravating factor. Reliability generally will fall, as will inspector's level of experience with the equipment in use. So old tricks (such as replacing the tachograph fuse with a blown one) will gain a new lease of life.

3. As noted above it seems that tachograph calibration may be tampered with given workshop cards, which contain only shared secrets and so should be widely forged. At present, intentional miscalibration can be proved if the setting within the instrument differs from that recorded on the plate, and the seal is intact; it is unclear what evidence could be extracted from the new digital system.

More generally, attacks on computer security systems involve the opportunistic exploitation of implementation defects, and these are more prevalent in new systems [1]. The introduction of digital tachographs will be no different: vehicle inspectors report increased tampering attempts whenever new technologies are introduced.

4. Finally, there will be a number of new avenues of attack which will appeal to the underground workshops that currently make their money from interruptors. One obvious target is the vehicle unit itself. The proposed regulations are silent on the anti-tampering mechanisms and standard of testing required here; but the move from an analogue tachograph to a digital one raises the possibility that tachographs' program code could be modified, or circuit boards substituted, or totally bogus devices manufactured which would conform with the regulations in all externally visible ways but contain extra features for the driver or operator. Criminals have already used altered or completely bogus cash machines and point-of-sale terminals in bank card fraud; bogus digital tachographs are to be expected.

The hope has been expressed that testing under the ITSEC programme will ensure that the equipment is fit for purpose. This may be the case eventually, but at present the institutions participating in ITSEC are oriented to evaluating 
military computer systems for NATO use. An early attempt to produce ITSEC testing criteria for smartcard-based systems yielded a document which emphasises the secrecy of the chip design [25] - a relatively pointless goal given that attackers can buy microscopes. Much more convincing test criteria have been developed by VISA [31].

\section{Conclusions}

It is often thought that introducing advanced technology such as smartcards and cryptography will make systems more secure. The Tachosmart proposals provide an interesting counterexample; there is consensus among experienced enforcement officers that they will make the problem worse (as was the case with the earlier move from mechanical to analogue electronic instruments).

The practical issues raised are important and urgent. Even with a good design, the costs of a changeover will be significant; the recent introduction of armoured tachograph cable cost many millions of ECU. Yet the design currently proposed does not address the most common abuses, and those that it does try to tackle are not dealt with effectively. Its introduction would ensure that much fraud will become almost impossible to detect, and that the regulations will fall into disrepute. The cost of such a failure could amount to thousands of additional traffic fatalities, and billions of ECU, before control could be restored.

We therefore present the problem of how to design a serviceable digital tachograph as a worthy problem to the research community.

Acknowledgements: A number of people provided valuable background information including Peter Dean, Department of Transport; Jean-Philippe Lelièvre, ERTICO; Gregory Clough, UCL; John Martin, Hampshire Constabulary; Gary Geldart, Vehicle Inspectorate; Dick Edmonds, Phil Needham and Nick Rendle of Lucas Kienzle; and Frank Clish of TVI. Roger Needham of Microsoft Research and Markus Kuhn of Cambridge University provided a sounding board as this analysis was developed.

\section{References}

1. RJ Anderson, "Why Cryptosystems Fail", in Proceedings of the 1st ACM Conference on Computer and Communications Security (November 1993) pp 215-227

2. RJ Anderson, MG Kuhn, "Tamper Resistance - a Cautionary Note", in The Second USENIX Workshop on Electronic Commerce Proceedings (Nov 1996) pp $1-11$

3. E Bovenlander, invited talk on smartcard security, Eurocrypt 97

4. 'Licensing of Trusted Third Parties for the Provision of Encryption Services', Department of Trade and Industry, March 1997

5. EU Regulation 3820/85 (drivers' rules) 
6. 'Council Regulation (EEC) no 3821/85 of 20 December 1985 on recording equipment in road transport' No L $370 / 8,31 / 12 / 85$

7. 'Draft Council Regulation Amending Councul Regulation (EEC) no 3821/85 and Councul Directive 88/599/EEC on Recording Equipment in Road Transport', EU, The Council, Interinstitutional file no 94/0187 (SYN), 2 July 1997

8. Euraxiat - Security Analysis, March 1997

9. "False Records: Analogue Tachographs", G Geldart, Vehicle Inspectorate, 22nd January 1998

10. Hampshire police training video

11. "Sleep related vehicle accidents", JA Horne, LA Reyner, in British Medical Journal $\mathrm{v} 310(4 / 3 / 95)$ pp 565-567

12. "Falling Asleep at the Wheel", J Horne, L Reyner, preprint, 29/2/96; distributed at Falling Asleep at the Wheel, Loughborough University 18-19/11/96

13. "Ex-radio chief 'masterminded' TV cards scam" Irish Independent 17/2/98

14. "Vulnerability Assessment of Security Seals", RG Johnson, ARE Garcia, in Journal of Security Administration v 20 no 1 (June 97) pp 15-27

15. 'Driver Card 16kByte version 00.01.01', 24/11/97

16. 'Workshop, Control and Company Cord version 00.01.01', 24/11/97

17. 'External Interface version 00.01.01', 25/11/97

18. 'Card Issuing version 00.02.00', 25/11/97

19. O Kocar, "Hardwaresicherheit von Mikrochips in Chipkarten", in Datenschutz und Datensicherheit v 20 no 7 (July 96) pp 421-424

20. "Falling Asleep at the Wheel", JS Martin, report on conference of the same name, Loughborough University 18-19/11/96

21. "Principal frauds used on the current tachographs", J Martin, UK response to ERTICO consultation document on tachograph falsification

22. 'Princıpales fraudes partant atteinte à l'ıntégrité du chronotachygraphe', Ministère de l'Équipement, des Transports et du Tourisme, Direction des Transports Terrestres, Sous-direction des transports routiers, bureau R3 (France)

23. "Denial of Service: An Example", RM Needham, in Communications of the ACM v 37 no 11 (Nov 94) pp 42-46

24. "Spying tags could stop fuel fraud", N Nuttall, in The Times (Interface Section) $28 / 1 / 98$ p 7

25. 'Common Criteria for IT Security Evaluation - Smartcard Integrated Circuit Protection Profile', registered at the French Certification Body under the number PP/9704; Motorola, Philips, Siemens, SGS-Thomson, Texas Instruments, October 1997.

26. "Driver sleepiness as a causal factor in accidents on the M180/A180 in South Humberside: A Preliminary Analysis", L Reyner, at Falling Asleep at the Wheel, Loughborough University 18-19/11/96

27. 'Tachosmart 3 Digital Tachograph - Final report', Thomson CSF, 1996

28. "Ministers unveil plans to toughen drink-drive laws", in The Times $2 / 2 / 98$ p 2

29. "Commission wants black box, smart cards to enforce road safety", A Torres, Reuters news item 0804 (2/9/94)

30. 'A Report on the Fitment of Tachograph Interruptors', 10/8/95, Vehicle Inspectorate, Wighill Lane, Walton, Wetherby, West Yorkshire

31. 'Integrated Circuit Chip Card - Security Gundelines Summary for IC Chip Design, Operating System and Application Design, Implementation Verification' v $2.1,4 / 11 / 97$ 